\title{
DIMENSION, GEOMETRIC, AGRICULTURAL AND QUALITY CHARACTERISTICS OF SAFFLOWER SEEDS
}

\author{
Rahim $A D A^{1 *}$ \\ ${ }^{1}$ Selcuk University, Faculty of Agriculture, Department of Field Crops, Konya, TURKEY \\ *Corresponding author: rahimada@selcuk.edu.tr
}

Received: 03.05.2013

\begin{abstract}
This research was carried out in the research field of Agricultural Faculty, Selcuk University, Konya, Turkey. Field experiments were carried out in two growing seasons (2011 and 2012) according to "Randomized Complete Block Design" with three replications. In the study, a total of 13 promised safflower (Carthamus tinctorius L.) lines (A13, A29, C12, E12, F4, F5, F6, G16, H3, J19, Y1-8-14-1, AOL-2(2), DP 1.5.8-1) and 5 cultivars (Black Sun1, KS 06 and Oleic Leed, Dinçer, Remzibey) were used as material. Oil content of the safflower genotypes were ranged in between $\mathbf{2 4 . 0 5 - 3 3 . 1 8 \%}$. The highest protein content value was obtained from J19 line $(\mathbf{2 1 . 7 2 \%})$ while the lowest was found on the Turkish cultivar of Remzibey (16.03\%). Negative correlations were found for oil content and hull thickness $(\mathbf{r}=-\mathbf{0 . 3 1 5 2} * *)$, hull ratio $(\mathbf{r}=-\mathbf{0 . 7 1 2 2} * *)$ and pappus ratio $(r=-0.3408 * *)$. Protein content was found as significantly and negative correlated with some characters, similar to the seed widness $(r=-\mathbf{0 . 2 5 4 6} * *)$, seed thickness $(r=-0.1918 *)$, geometric diameter $(r=-0.3194 * *)$, seed surface area $(r=-0.3119 * *)$, pappus ratio $(r=-0.3114 *)$ and 100 -seed weight $(r=-0.2597)$, respectively. According to the results, increasing the dimension and geometrical values cause to a decrease in the oil and protein content of safflower seeds. Quality components (oil and protein content) showed negative correlations with both of pappus rate and hull features.
\end{abstract}

Keywords: Carthamus tinctorius, dimension, hull, oil, protein, Safflower seed

\section{INTRODUCTION}

Safflower is a member of the family Asteraceae, it is cultivated primarily for achene (seed), which is used as oil and birdseed. Flowers are known to have many medical properties (Dajue and Mundel, 1996) and cut flowers (Uter, 2008). The leaves, shoots of safflower are also used for salad (Nimbkar, 2002).

Number of drought resistant crops such as safflower should be increased over the world. (Singh and Nimbkar, 2007). Although Turkey is not a big part in the world safflower seed production, areas under safflower seed cultivation have recently increased (Sacilik et al., 2007).

In the former researches, safflower breeders typically focused on the yield, quality and resistance breeding, etc. characteristics (Urie and Zimmer, 1970; Urie, 1986; Singh et al., 2008; Ada, 2012). However, there is necessary to improvement of the genotypes which are convenient to the demand of industry. According to Tarighi et al. (2010), it is necessary to understand the physical features of safflower in order to develop equipment for sowing, harvesting, and storage and oil extraction of safflower seeds. The dimensions of safflower are important in design of separating and grinding in the machines.
Researchers (Sadeghi et al., 2011; Kaya et al., 2011) put forth interesting results about the relationship between dimension and seed germination properties. Therefore, the best way to know the properties of safflower seeds has multiple benefits. This study was in Konya-Turkey conditions during the both years of 2011 and 2012 and, the seed characteristics of the safflower cultivars and lines were determined.

\section{MATERIALS AND METHOD}

In the study, a total of 13 safflower promised lines (A13, A29, C12, E12, F4, F5, F6, G16, H3, J19, Y1-8-141) which were selected and collected from Konya natural vegetation and 2 lines (AOL-2(2), DP 1.5.8-1) obtained by pedigree method and breeded by Dr. Rahim ADA, and 3 American cultivars (Black Sun1, KS 06 and Oleic Leed), 2 Turkey cultivars (Dinçer, Remzibey) were used, seeds were produced in the research field of Agricultural Faculty, Selcuk University, Konya, Turkey in two growing seasons (2011 and 2012). The study was conducted on $4^{\text {th }}$ of April 2011 and $05^{\text {th }}$ of April 2012 according to "Randomized Complete Block Design" with three replications. Each genotype was sown in plots with 4 rows, $4 \mathrm{~m}$ of longitude with spacing $50 \mathrm{~cm}$ between rows. 
In the both years of 2011 and 2012 the amounts of total precipitations between April and August were 201.3 and $66.8 \mathrm{~mm}$ respectively. Average temperature was $18.3^{\circ} \mathrm{C}$ and $20.6^{\circ} \mathrm{C}$ in the first and the second vegetation

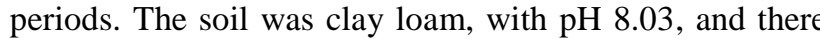
was not any salinity problem.

In the experiments, a total of $40 \mathrm{~kg} \mathrm{ha}^{-1}$ of $\mathrm{P}_{2} \mathrm{O}_{5}$ and 30 $\mathrm{kg} \mathrm{ha}^{-1}$ of nitrogen were applied before sowing and $20 \mathrm{~kg}$ $\mathrm{ha}^{-1}$ of nitrogen (ammonium nitrate $33 \%$ ) was used as a top fertilizing during starting of stem elongation. Weeds were controlled by hand.

The seeds were cleaned manually to remove all foreign matter such as chaff as well as immature and broken seeds. The three linear dimension of the seeds (Figure 1), namely length (L), widness (W) and thickness (T) were measured using a micrometer $(0.01 \mathrm{~mm}$ of sensitivity) (Baumler et al., 2006).

Geometric mean diameter (Dg) and sphericity (Ø) values were found using the following formula (Mohsenin, 1970; Calisir et al., 2005):

$$
\begin{aligned}
& \text { Dg }=(\mathrm{LWT})^{1 / 3} \\
& \varnothing=(\mathrm{LWT})^{1 / 3} / \mathrm{L}
\end{aligned}
$$

where: $\mathrm{L}$ is the length, $\mathrm{W}$ is the widness and $\mathrm{T}$ is the thickness, all the data were recorded as mm unit.

The seed surface area of safflower was found by analogy with a sphere of same geometric mean diameter, using the following expression cited by Sacilik et al. (2003).

\section{$\mathrm{S}=\pi \mathrm{Dg}$}

where $s$ is the surface area in $\mathrm{mm}^{2}$ and $\mathrm{Dg}$ is the geometric mean diameter in $\mathrm{mm}$.

Hull percentage, oil (Soxhlet) and protein (Kjeldahl) analysis made according to Keles (2010).

Analysis of correlation (JUMP, SAS Institute Inc. 1989-2002) and variance (MSTAT-C, Michigan State University, 1983) were performed using statistical software packages.
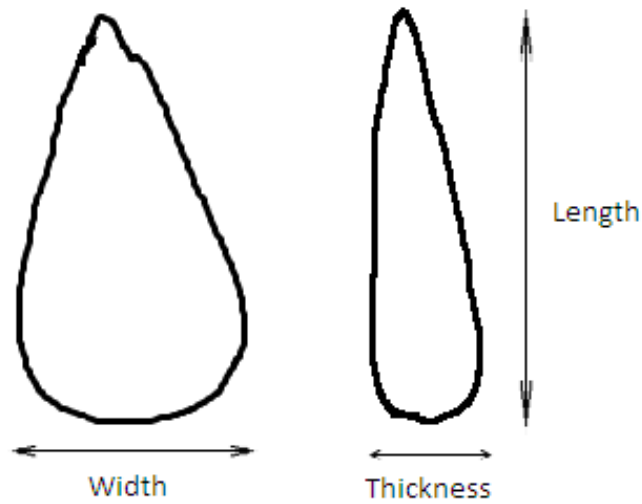

Figure 1. Linear dimension of safflower

\section{RESULTS}

The differences among the genotypes were significant for all seed characteristics (Table 1 and 2).

\begin{tabular}{|c|c|c|c|c|c|c|c|c|c|c|c|c|}
\hline $\begin{array}{l}\text { Genotypes } \\
\text { of } \\
\text { safflower }\end{array}$ & $\begin{array}{l}\text { Seed } \\
\text { Length } \\
(\mathrm{mm})\end{array}$ & $\begin{array}{l}\text { Seed } \\
\text { Widness } \\
(\mathbf{m m})\end{array}$ & $\begin{array}{l}\text { Seed } \\
\text { Thickness } \\
(\mathrm{mm})\end{array}$ & $\begin{array}{l}\text { Geometric } \\
\text { Mean } \\
\text { Diameter } \\
(\mathbf{m m})\end{array}$ & $\begin{array}{l}\text { Sphericity } \\
(\%)\end{array}$ & $\begin{array}{l}\text { Seed } \\
\text { Surface } \\
\text { Area } \\
\left(\mathrm{mm}^{2}\right) \\
\end{array}$ & $\begin{array}{l}\text { Hull } \\
\text { Thickness } \\
(\mathrm{mm})\end{array}$ & $\begin{array}{l}\text { Hull } \\
\text { Ratio } \\
(\%)\end{array}$ & $\begin{array}{l}\text { Pappus } \\
\text { Ratio } \\
(\%)\end{array}$ & $\begin{array}{l}\text { 100- } \\
\text { Seed } \\
\text { Weight } \\
\text { (g) } \\
\end{array}$ & $\begin{array}{l}\text { Oil } \\
\text { Content } \\
(\%)\end{array}$ & $\begin{array}{l}\text { Protein } \\
\text { Content } \\
(\%)\end{array}$ \\
\hline Dinçer & $7.27^{\mathrm{a}-\mathrm{d}}$ & $3.78^{\mathrm{cd}}$ & $3.14^{\mathrm{fg}}$ & $4.42^{\mathrm{e}-\mathrm{h}}$ & $0.607^{\text {ef }}$ & $61.24^{\text {efg }}$ & $0.397^{\mathrm{ab}}$ & $51.37^{\mathrm{c}-\mathrm{g}}$ & $1.45^{\mathrm{e}}$ & $3.63^{\mathrm{ef}}$ & $26.29^{\mathrm{efg}}$ & $17.17^{\mathrm{e}-\mathrm{h}}$ \\
\hline Remzibey & $6.86^{\mathrm{b}-\mathrm{e}}$ & $3.97^{\mathrm{bcd}}$ & $3.25^{\mathrm{d}-\mathrm{g}}$ & $4.46^{\mathrm{b}-\mathrm{h}}$ & $0.650^{\mathrm{bcd}}$ & $62.38^{\mathrm{c}-\mathrm{g}}$ & $0.362^{\mathrm{abc}}$ & $48.49^{\text {fgh }}$ & $10.62^{\mathrm{abc}}$ & $3.36^{\mathrm{fgh}}$ & $29.30^{\text {cd }}$ & $16.03^{\mathrm{h}}$ \\
\hline Black & $7.10^{\mathrm{a}-\mathrm{e}}$ & $3.91^{\mathrm{bcd}}$ & $3.21^{\mathrm{efg}}$ & $4.46^{\mathrm{b}-\mathrm{h}}$ & $0.629^{\text {cde }}$ & $62.54^{\mathrm{b}-\mathrm{g}}$ & $0.357^{\text {bcd }}$ & $43.39^{\mathrm{ij}}$ & $1.66^{\mathrm{de}}$ & $3.42^{\text {fgh }}$ & $31.44^{\mathrm{ab}}$ & $19.53^{\mathrm{bc}}$ \\
\hline \multicolumn{13}{|l|}{ Sun1 } \\
\hline KS 06 & $7.54^{\mathrm{ab}}$ & $4.14^{\mathrm{bc}}$ & $3.27^{\mathrm{c}-\mathrm{g}}$ & $4.68^{\mathrm{a}-\mathrm{d}}$ & $0.621^{\text {cde }}$ & $68.85^{\mathrm{a}-\mathrm{e}}$ & $0.353^{\mathrm{bcd}}$ & $42.74^{\mathrm{i}}$ & $2.06^{\mathrm{de}}$ & $4.02^{\mathrm{abc}}$ & $33.18^{\mathrm{a}}$ & $17.30^{\mathrm{e}-\mathrm{h}}$ \\
\hline Oleic Leed & $6.51^{\mathrm{e}}$ & $3.78^{\mathrm{cd}}$ & $3.29^{\mathrm{b}-\mathrm{g}}$ & $4.32^{\text {fgh }}$ & $0.665^{\mathrm{ab}}$ & $58.73^{\mathrm{fg}}$ & $0.315^{\mathrm{d}}$ & $40.51^{\mathrm{j}}$ & $1.15^{\mathrm{e}}$ & $3.26^{\mathrm{gh}}$ & $32.47^{\mathrm{a}}$ & $19.32^{\mathrm{bc}}$ \\
\hline A13 & $7.40^{\mathrm{abc}}$ & $4.06^{\mathrm{bcd}}$ & $3.63^{\mathrm{a}}$ & $4.77^{\mathrm{a}}$ & $0.646^{\mathrm{bcd}}$ & $71.68^{\mathrm{a}}$ & $0.361^{\mathrm{abc}}$ & $52.52^{\mathrm{b}-\mathrm{e}}$ & $14.50^{\mathrm{ab}}$ & $4.15^{\mathrm{a}}$ & $26.28^{\mathrm{efg}}$ & $17.55^{\mathrm{d}-\mathrm{g}}$ \\
\hline A29 & $7.49^{\mathrm{abc}}$ & $4.07^{\mathrm{bcd}}$ & $3.43^{\text {a-e }}$ & $4.71^{\mathrm{ab}}$ & $0.629^{\text {cde }}$ & $69.74^{\mathrm{a}-\mathrm{d}}$ & $0.385^{\mathrm{ab}}$ & $53.65^{\text {bcd }}$ & $16.42^{\mathrm{a}}$ & $4.10^{\mathrm{ab}}$ & $25.91^{\mathrm{f}-\mathrm{i}}$ & $17.91^{\text {def }}$ \\
\hline C12 & $7.07^{\mathrm{a}-\mathrm{e}}$ & $4.33^{\mathrm{ab}}$ & $3.47^{\mathrm{a}-\mathrm{d}}$ & $4.73^{\mathrm{a}}$ & $0.670^{\mathrm{ab}}$ & $70.34^{\mathrm{ab}}$ & $0.397^{\mathrm{ab}}$ & $55.39^{\mathrm{ab}}$ & $10.57^{\mathrm{bc}}$ & $3.79^{b-e}$ & $24.86^{\text {ghi }}$ & $17.16^{\mathrm{e}-\mathrm{h}}$ \\
\hline E12 & $6.97^{\mathrm{a}-\mathrm{e}}$ & $4.03^{\text {bcd }}$ & $3.31^{\mathrm{b}-\mathrm{g}}$ & $4.53^{\mathrm{a}-\mathrm{g}}$ & $0.650^{\text {bcd }}$ & $64.30^{\mathrm{a}-\mathrm{g}}$ & $0.328^{\mathrm{cd}}$ & $47.27^{\mathrm{hi}}$ & $5.27^{\text {cde }}$ & $3.18^{\mathrm{h}}$ & $30.33^{\mathrm{bc}}$ & $16.39^{\mathrm{gh}}$ \\
\hline
\end{tabular}

Table 1. Some seed characteristics of safflower genotypes (2011 and 2012)

The seed length, widness and thickness of the used genotypes are shown in the Table 1 and 2 show. The highest value for seed length $(7.64 \mathrm{~mm})$, widness $(4.73$ $\mathrm{mm})$ and thickness $(3.63 \mathrm{~mm})$ were observed at J19, F4 and A13 safflower lines. The lowest seed length, widness and thickness were recorded from Oleic Leed $(6.51 \mathrm{~mm})$, AOL-2(2) (3.57 mm) and H3 (3.09 mm).

There were significant differences among different safflower genotypes in terms of the parameters of geometric mean diameter, sphericity and seed surface area. Geometric mean diameter, sphericity and seed surface area were ranged from $4.25-4.77 \mathrm{~mm}, 0.589$ $0.693 \%$ and $56.82-71.68 \mathrm{~mm}^{2}$ respectively on safflower genotypes.

The highest hull thickness and ratio in the safflower genotypes were obtained from H3 (0.402 mm) and F4 $(58.52 \%)$ lines, whereas Oleic Leed cultivar $(0.315 \mathrm{~mm}$ and $40.51 \% \%$ ) resulted with the lowest values. 
Table 2. Some seed characteristics of safflower genotypes (2011 and 2012) (continue)

\begin{tabular}{|c|c|c|c|c|c|c|c|c|c|c|c|c|}
\hline $\begin{array}{l}\text { Genotyp } \\
\text { es of } \\
\text { safflower }\end{array}$ & $\begin{array}{l}\text { Seed } \\
\text { Length } \\
(\mathrm{mm})\end{array}$ & $\begin{array}{l}\text { Seed } \\
\text { Widnes } \\
\text { s (mm) }\end{array}$ & $\begin{array}{l}\text { Seed } \\
\text { Thicknes } \\
\text { s (mm) }\end{array}$ & $\begin{array}{l}\text { Geometri } \\
\text { c Mean } \\
\text { Diameter } \\
(\mathrm{mm})\end{array}$ & $\begin{array}{l}\text { Sphericity } \\
(\%)\end{array}$ & $\begin{array}{l}\text { Seed } \\
\text { Surfac } \\
\text { e Area } \\
\left(\mathbf{m m}^{2}\right) \\
\end{array}$ & $\begin{array}{l}\text { Hull } \\
\text { Thicknes } \\
\text { s (mm) }\end{array}$ & $\begin{array}{l}\text { Hull } \\
\text { Ratio } \\
(\%)\end{array}$ & $\begin{array}{l}\text { Pappus } \\
\text { Ratio } \\
(\%)\end{array}$ & $\begin{array}{l}\text { 100-Seed } \\
\text { Weight } \\
\text { (g) }\end{array}$ & $\begin{array}{l}\text { Oil } \\
\text { Content } \\
(\%)\end{array}$ & $\begin{array}{l}\text { Protein } \\
\text { Content } \\
(\%)\end{array}$ \\
\hline F4 & $6.79^{\text {cde }}$ & $4.73^{\mathrm{a}}$ & $3.34^{\mathrm{b}-\mathrm{g}}$ & $4.70^{\mathrm{abc}}$ & $0.693^{\mathrm{a}}$ & $69.98^{\mathrm{abc}}$ & $0.381^{\mathrm{ab}}$ & $58.52^{\mathrm{a}}$ & $10.55^{\mathrm{bc}}$ & $3.41^{\text {fgh }}$ & $24.36^{\mathrm{hi}}$ & $16.98^{\mathrm{fgh}}$ \\
\hline F5 & $7.03^{\mathrm{a}-\mathrm{e}}$ & $4.12^{\mathrm{bc}}$ & $3.51^{\mathrm{abc}}$ & $4.66^{\mathrm{a}-\mathrm{e}}$ & $0.664^{\mathrm{ab}}$ & $68.35^{\mathrm{a}-\mathrm{d}}$ & $0.368^{\mathrm{abc}}$ & $49.48^{\mathrm{e}-\mathrm{h}}$ & $5.15^{\text {cde }}$ & $3.86^{\mathrm{a}-\mathrm{e}}$ & $27.99^{\mathrm{de}}$ & $16.72^{\mathrm{fgh}}$ \\
\hline F6 & $7.11^{\mathrm{a}-\mathrm{e}}$ & $4.05^{\mathrm{bcd}}$ & $3.53^{\mathrm{ab}}$ & $4.66^{\mathrm{a}-\mathrm{e}}$ & $0.656^{\mathrm{bc}}$ & $68.22^{\mathrm{a}-\mathrm{e}}$ & $0.388^{\mathrm{ab}}$ & $51.55^{\mathrm{b}-\mathrm{g}}$ & $4.65^{\mathrm{de}}$ & $3.56^{\text {efg }}$ & $24.95^{\text {ghi }}$ & $18.27^{\text {cde }}$ \\
\hline G16 & $7.05^{\mathrm{a}-\mathrm{e}}$ & $3.87^{\mathrm{bcd}}$ & $3.23^{\mathrm{d}-\mathrm{g}}$ & $4.45^{\mathrm{ch}}$ & $0.632^{\mathrm{b}-\mathrm{e}}$ & $62.27^{\mathrm{c}-\mathrm{g}}$ & $0.388^{\mathrm{ab}}$ & $52.35^{\mathrm{b}-\mathrm{f}}$ & $6.80^{\text {cde }}$ & $3.40^{\text {fgh }}$ & $27.16^{\text {ef }}$ & $16.66^{\mathrm{fgh}}$ \\
\hline $\mathrm{H} 3$ & $6.61^{\mathrm{d}-\mathrm{e}}$ & $3.77^{\mathrm{cd}}$ & $3.09^{\mathrm{g}}$ & $4.25^{\mathrm{h}}$ & $0.643^{\mathrm{bcd}}$ & $56.82^{\mathrm{g}}$ & $0.402^{\mathrm{a}}$ & $52.67^{\mathrm{b}-\mathrm{e}}$ & $3.30^{\text {de }}$ & $3.21^{\mathrm{h}}$ & $24.08^{\mathrm{i}}$ & $21.72^{\mathrm{a}}$ \\
\hline J19 & $7.64^{\mathrm{a}}$ & $4.02^{\text {bcd }}$ & $3.39^{\mathrm{a}-\mathrm{f}}$ & $4.71^{\mathrm{ab}}$ & $0.617^{\mathrm{def}}$ & $69.58^{\mathrm{a}-\mathrm{d}}$ & $0.368^{\mathrm{abc}}$ & $50.48^{\mathrm{d}-\mathrm{h}}$ & $6.88^{\text {cde }}$ & $3.95^{\mathrm{a}-\mathrm{d}}$ & $26.58^{\mathrm{efg}}$ & $18.59^{\mathrm{bcd}}$ \\
\hline $\begin{array}{l}\text { Y1-8-14- } \\
1\end{array}$ & $6.65^{\mathrm{de}}$ & $3.74^{\mathrm{cd}}$ & $3.18^{\mathrm{efg}}$ & $4.29^{\mathrm{gh}}$ & $0.646^{\mathrm{bcd}}$ & $57.80^{\mathrm{f}-\mathrm{g}}$ & $0.391^{\mathrm{ab}}$ & $55.18^{\mathrm{abc}}$ & $7.41^{\mathrm{cd}}$ & $2.66^{\mathrm{i}}$ & $26.20^{\mathrm{e}-\mathrm{h}}$ & $19.22^{\mathrm{bc}}$ \\
\hline $\mathrm{AOL}-2(2)$ & $7.54^{\mathrm{ab}}$ & $3.57^{\mathrm{d}}$ & $3.25^{\mathrm{d}-\mathrm{g}}$ & $4.44^{\mathrm{d}-\mathrm{h}}$ & $0.589^{f}$ & $61.88^{\mathrm{d}-\mathrm{g}}$ & $0.388^{\mathrm{ab}}$ & $47.70^{\mathrm{gh}}$ & $2.63^{\mathrm{de}}$ & $3.78^{\text {cde }}$ & $31.76^{\mathrm{ab}}$ & $19.60^{\mathrm{b}}$ \\
\hline $\begin{array}{l}\text { DP } 15-8- \\
1\end{array}$ & $6.99^{\mathrm{a}-\mathrm{e}}$ & $4.08^{\text {bcd }}$ & $3.30^{\mathrm{b}-\mathrm{g}}$ & $4.55^{\mathrm{a}-\mathrm{f}}$ & $0.651^{\mathrm{bc}}$ & $65.15^{\mathrm{a}-\mathrm{f}}$ & $0.354^{\text {bcd }}$ & $49.24^{\mathrm{efg}}$ & $1.45^{\mathrm{e}}$ & $3.65^{\text {def }}$ & $27.85^{\mathrm{de}}$ & $18.39^{\mathrm{b}-\mathrm{e}}$ \\
\hline Mean & 7.10 & 3.99 & 3.33 & 4.55 & 0.642 & 64.99 & 0.371 & 50.14 & 6.25 & 3.58 & 27.83 & 18.02 \\
\hline Mean & $0.68194 *$ & 0.39838 & $0.12513^{*}$ & $0.16345^{*}$ & 0.00354 ** & 134.33 & $0.00353^{*}$ & $129.701 *$ & $129.501^{*}$ & $0.87228^{*}$ & 50.6486* & $12.5621 *$ \\
\hline Square & $*$ & * & * & $*$ & & $6 * *$ & & $*$ & $*$ & $*$ & * & $*$ \\
\hline $\begin{array}{l}\text { LSD } \\
\text { Value }\end{array}$ & 0.7186 & 0.5411 & 0.2511 & 0.2586 & 0.03871 & 7.908 & 0.04746 & 3.953 & 5.814 & 0.3174 & 1.841 & 1.283 \\
\hline
\end{tabular}

With respect to pappus ratio, the A 29 line (16.42\%) had the highest value, while the lowest value were obtained from Oleic leed $(1.15 \%)$, Dinçer $(1.45 \%)$ cultivar and DP $15-8-1(1.45 \%)$ line.

100 -seed weight of safflower genotypes were ranged from 2.66 to $4.15 \mathrm{~g}$. The highest 100 -seed weight was recorded from A13 line, whereas the lowest 100-seed weight was recorded from Y1-8-14-1.

Safflower genotypes had significant effect on the oil content $(\mathrm{p}<0.01)$. Oil ratios were varied from $24.05 \%$ to $33.18 \%$. America originated cultivars were in the same group as statically; KS $06(33.18 \%)$ and Oleic Leed $(32.47 \%)$ had the highest oil content, whereas H3 $(24.08 \%)$ had the lowest ratio.

Differences among the safflower genotypes were found as significant for protein content. The highest protein content value was obtained from H3 line (21.72\%) while the lowest was found from the Turkish cultivar Remzibey (16.03\%).

Correlation coefficients among the studied safflower seed characteristics were given in Table 3, 4 and 5 . Negative correlations were found among oil content and hull thickness ( $\mathrm{r}=-0.3152 * *)$, hull ratio $\left(\mathrm{r}=-0.7122^{*}\right)$ and pappus ratio $(\mathrm{r}=-0.3408 * *)$ (Table 4$)$.

Protein content was significant but negatively correlated with the fallowing characters; seed widness ( $\mathrm{r}=$ $\left.-0.2546^{* *}\right)$, seed thickness ( $\left.\mathrm{r}=-0.1918^{*}\right)$, geometric mean diameter $(\mathrm{r}=-0.3194 * *)$, seed surface area $\left(\mathrm{r}=-0.3119^{* *}\right)$, pappus ratio $\left(\mathrm{r}=-0.3114^{* *}\right)$ and 100 -seed weight $(\mathrm{r}=-$ 0.2597 ), respectively (Table 5).

\section{DISCUSSION}

Oil content of achene (seed) is a very important economic character for safflower genotypes and considered one of the most important factors affecting the success of safflower production in different regions (Vorpsi et al., 2010). Similarly, the oil-cake meal resulting from pressing the oil from the seed is being offered as a feed for livestock under the name whole pressed safflower seed meal (Goss and Otogaki, 1954). Therefore, plant breeders should focus on the physical properties of safflower seeds which are adapted to the design of equipment for handling, storing, dehulling and processing. These safflower properties are affected by numerous factors such as size and form features of the achene (seed) (Baumler et al., 2006).

Earlier studies which were investigated for different humidity levels seed length, widness, thickness and geometric mean diameter and sphericity were reported as it seen in the fallowing line: Aktas et al. (2006) found 7.27-7.81 mm, 3.50-3.79 mm, 2.80-3.50 mm, 4.46-4.84 $\mathrm{mm}$ and 47.14-48.83\% respectively, Calisir et al. (2005) 6.89-7.56 mm, 3.76-4.36 mm, 2.71-3.19 mm, 4.13-4.70 $\mathrm{mm}$ and 60.00-62.30\% respectively and seed surface area was found by Seifi et al. (2010) as a value of 65.63-79.15 $\mathrm{mm}^{2}$, Tarighi et al., (2010) reported as 57.2-70.38 $\mathrm{mm}^{2}$ and Tarighi et al. (2011) found $62.77-72.38 \mathrm{~mm}^{2}$. It is well known that there is a positive correlation between 100 -seed weight and seed yield in safflower (Singh et al., 2008). This feature (seed yield) is quite important for manufacturers. In terms of 100-seed weight, a similar result with the present research was reported by Arslan et al. (2008) with a range from $3.86 \mathrm{~g}$ to $4.82 \mathrm{~g}$. 
Table 3. Correlation coefficients of the seed characteristics

\begin{tabular}{|c|c|c|c|c|}
\hline Variable & by Variable & Correlation & & \\
\hline Seed Widness & Seed Length & 0,0487 & & $\square$ \\
\hline Seed Thickness & Seed Length & $0,2029 *$ & & $\square$ \\
\hline Seed Thickness & Seed Widness & $0,2087 *$ & & P\&\& \\
\hline Geometric Mean Diameter & Seed Length & $0,5089 * *$ & & \\
\hline Geometric Mean Diameter & Seed Widness & $0,7706^{* *}$ & & \%భ\%\&\$\%\& \\
\hline Geometric Mean Diameter & Seed Thickness & $0,6537 * *$ & & \\
\hline Sphericity & Seed Length & $-0,5533 * *$ & ᄃ & $\square$ \\
\hline Sphericity & Seed Widness & $0,7057 * *$ & & 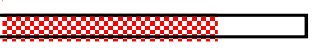 \\
\hline Sphericity & Seed Thickness & $0,4068 * *$ & & \&\$\& \\
\hline Sphericity & Geometric Mean Diameter & $0,4340 * *$ & & \\
\hline Seed Surface Area & Seed Length & $0,4832 * *$ & & 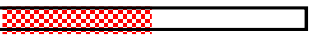 \\
\hline Seed Surface Area & Seed Widness & $0,7975^{* *}$ & & \\
\hline Seed Surface Area & Seed Thickness & $0,6385^{* *}$ & & 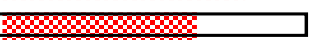 \\
\hline Seed Surface Area & Geometric Mean Diameter & $0,9984 * *$ & & 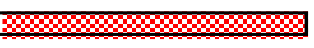 \\
\hline Seed Surface Area & Sphericity & $0,4606 * *$ & & \\
\hline Hull Thickness & Seed Length & 0,0240 & & 3 \\
\hline Hull Thickness & Seed Widness & 0,0496 & & 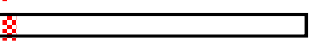 \\
\hline Hull Thickness & Seed Thickness & $-0,0313$ & & \\
\hline Hull Thickness & Geometric Mean Diameter & 0,0215 & & \\
\hline Hull Thickness & Sphericity & $-0,0077$ & & $\sqsupset$ \\
\hline Hull Thickness & Seed Surface Area & 0,0271 & & 3 \\
\hline Hull Ratio & Seed Length & $-0,0695$ & & \\
\hline Hull Ratio & Seed Widness & 0,0973 & & \\
\hline Hull Ratio & Seed Thickness & 0,0088 & & \\
\hline Hull Ratio & Geometric Mean Diameter & 0,0407 & $\square$ & 3 \\
\hline Hull Ratio & Sphericity & 0,1112 & $\square$ & $\notin$ \\
\hline Hull Ratio & Seed Surface Area & 0,0477 & $\square_{2}$ & 8 \\
\hline
\end{tabular}

Table 4. Correlation coefficients of the seed characteristics (continue)

\begin{tabular}{|c|c|c|}
\hline Variable & by Variable & Correlation \\
\hline Hull Ratio & Hull Thickness & $0,2967 * * \square$ \\
\hline Pappus Ratio & Seed Length & $0,1408 \square$ \\
\hline Pappus Ratio & Seed Widness & $0,3161 * * \square$ \\
\hline Pappus Ratio & Seed Thickness & $0,3421 * * \square$ \\
\hline Pappus Ratio & Geometric Mean Diameter & $0,4086^{* *} \square$ \\
\hline Pappus Ratio & Sphericity & $0,2412 * \square$ \\
\hline Pappus Ratio & Seed Surface Area & $0,4166 * * \square$ \\
\hline Pappus Ratio & Hull Thickness & $0,1021 \square$ \\
\hline Pappus Ratio & Hull Ratio & $0,3763 * * \square$ \\
\hline 100-Seed Weight & Seed Length & $0,6316 * * \square$ \\
\hline 100-Seed Weight & Seed Widness & $0,2591 * * \square$ \\
\hline 100-Seed Weight & Seed Thickness & $0,3730 * * \square \square$ \\
\hline 100-Seed Weight & Geometric Mean Diameter & $0,5927 * * \square$ \\
\hline 100-Seed Weight & Sphericity & $-0,0948 \square$ \\
\hline 100-Seed Weight & Seed Surface Area & $0,5740 * * \square$ \\
\hline 100-Seed Weight & Hull Thickness & $0,1213 \square$ \\
\hline 100-seed weight & Hull Ratio & $-0,0880 \square$ \\
\hline 100-Seed Weight & Pappus Ratio & $0,1746 \square$ \\
\hline Oil Content & Seed Length & $0,0857 \square$ \\
\hline Oil Content & Seed Widness & $-0,0745 \square$ \\
\hline Oil Content & Seed Thickness & $-0,1627 \square$ \\
\hline Oil Content & Geometric Mean Diameter & $-0,0931 \square$ \\
\hline Oil Content & Sphericity & $-0,1722 \square$ \\
\hline Oil Content & Seed Surface Area & $-0,0955 \square$ \\
\hline Oil Content & Hull Thickness & $-0,3152 * * \square$ \\
\hline Oil Content & Hull Ratio & 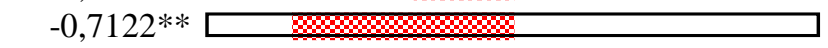 \\
\hline Oil Content & Pappus Ratio & $-0,3408 * * \quad \square+\frac{1}{\square}$ \\
\hline
\end{tabular}


Table 5. Correlation coefficients of the seed characteristics (continue)

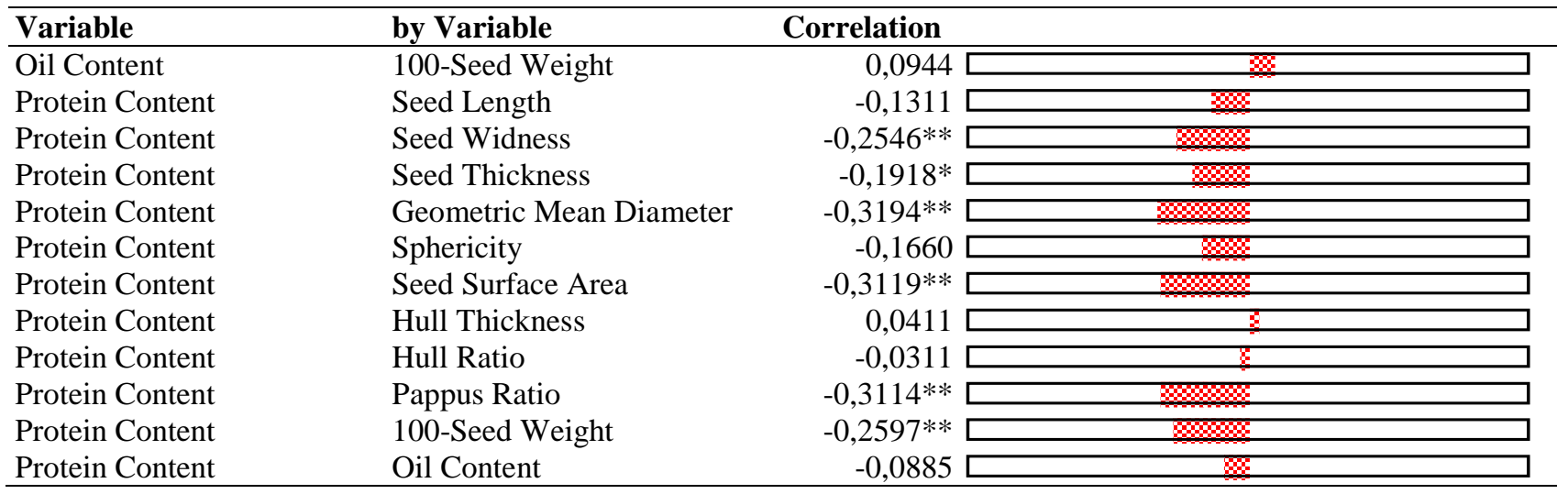

Some of the safflower genotypes had pappus but, this characteristic is not a desirable (Dajue and Mundel, 1996). In this study, while pappus percentage especially in developed safflower lines with a selection of natural vegetation varied between $3: 30$ to $16: 42 \%$, commercial cultivars determined between 1.15 and $10.62 \%$.

The oil content in the released genotypes was varying from 28 to $30 \%$, which needs an increase of 5-8\% in this crop (Yadava et al., 2012). In order to increase the content of oil seeds, plant breeders have attempted to reduce the testa tissue through breeding selection. Moreover, knowledge of characteristics of the seed testa is imperative. For this purpose, the reduction of sclerenchyma varies from seed to seed (Urie and Zimmer, 1970).

Baumler et al. (2006) worked on hull thickness and they reported the results between 0.282-0.407 values in different humidity levels. A well-developed hull (achene) cause to a reduction of $30 \%$ in oil content. Thickness of hull should be less than $50 \%$ in safflowers (Dajue and Mundel, 1996). Therefore, as the thick Hull tends to keep the oil content in safflower low, reduction of the Hull increases oil percentages (Dajue and Mundel, 1996) and meal quality (Urie and Zimmer, 1970).

Percentage of the Hull is an important component affecting the oil content (Weiss, 2000; Baumler et al., 2006) and environmental and other non-genetic factors may influence Hull content (Urie 1986). According to De Silva and Gordon (1986), if a high negative genotypic correlation exists between hull content and susceptibility, it may be difficult to select for recombinants favorable in both traits. Oil content of safflower genotypes varies between $54.3 \%$ and $58.4 \%$. Uysal et al (2006) found these values as $52.0-54.2 \%$, Keles and Ozturk (2012) found as $38.26-45.31 \%$. Safflower seeds are rich of protein content, hence it is valuable in animal feeding. For this reason, protein content in safflower is one of the important quality criteria.

The protein values obtained in this study gave similar results with the reports of Doğan and Serinc (1990) (17.62-24.22\%), Keles and Ozturk (2012) (17.13-19.76\%) and also Ada (2012) 24.04-28.79\%.

\section{CONCLUSION}

According to the results, increasing in the dimension and geometrical values caused to decreasing in the content of oil and protein in the safflower seeds. Pappus rate and quality requirements (oil and protein content) showed a negative correlation while, there was a positive correlation between hull characteristics and pappus.

Consequently, plant breeders should focus on the mentioned characteristics and pappus for seed selection.

\section{LITERATURE CITED}

Ada, R., 2012. Effects of winter and spring sowing on yield components of safflower Genotypes. World Academy of Science, Engineering and Technology 66: 622-626.

Aktas, T., I. Celen and R. Durgut, 2006. Some physical and mechanical properties of safflower seed (Carthamus tinctorius L.). Journal of Agronomy, 5(4): 613-616.

Arslan, B., E. Esendal and C. Pasa, 2008. The economically important traits of safflower (Carthamus tinctorius L.) cultivars and lines cultivated in Tekirdag, Turkey. Proceedings of the 7th International Safflower Conference, Wagga Wagga, New South Wales, Australia.

Baumler, E., A. Cuniberti, S. M. Nolasco and I. C. Riccobene, 2006. Moisture dependent physical and compression properties of safflower seed. Journal of Food Engineering, 72: $134-140$

Calisir, S., M. Ozcan, H. Haciseferogullari and M. U. Yildiz, 2005. A study on some physico-chemical properties of Turkey okra (Hibiscus esculenta L.) seeds. Journal of Food Engineering, 68: 73-78.

De Silva, H. N. and I. L. Gordan, 1986. Variance component in safflower (Carthamus tinctorius) trials in the Manawatu district, withthe implication for testing methods. New Zealand Journal of Experimentel Agriculture, 14:1-6.

Dajue, L. and H. H. Mundel, 1996. Safflower. Carthamus tinctorius L. Promoting the conservation and use of underutilized and neglected crops. 7. Institute of Plant Genetic and Crop Plant Research, Gatersleben / International Plant Genetic Resources Institute, Rome. Italy, 83 pp.

Dogan, A. and M. Serinc,, 1990. Studies on the oil quality of some selected safflower (Carthamus tinctorius L.) lines. Gida, 15(1): 51-56 (in Turkish).

Goss, H. and K. K. Otagaki, 1954. Safflower meal digestion tests, lambs used in digestion trials with decorticated seed meal to test product as feed for livestock. California Agriculture, page 15 . 
JUMP, 1989-2002. 5.0.1a: A Business Unit of SAS Copyright, SAS Institute Inc. http://www.jmp.com

Kaya, M., S. Bayramin, G. Kaya and O, Uzun, 2011. Seed vigor and ion toxicity in safflower (Carthamus tinctorius L.) seedlings produced by various dimensions under $\mathrm{NaCl}$ stress. Arch. Biol. Sci., Belgrade, 63 (3), 723-729.

Keles, R., 2010. Effects of different sowing dates on the yield, yield components and quality on some safflower (Carthamus tinctorius L.) cultivars. Selcuk University, Master Thesis.

Keles, R. and O. Ozturk, 2012. Effect of different sowing dates on the seed yield and quality of safflower. TABAD, 5 (1): 112-117.

Michigan State University, 1983. MSTAT-C: Micro- computer Statistical Program, Version 2.0. Michigan State University, East Lansing.

Mohsenin N.N., 1970. Physical properties of plant and animal materials. Gordon and Breach Science Publishers, Inc., New York.

Nimbkar, N., 2002. Safflower rediscovered. Times Agric. J. 2: 32-36.

Sacilik, K., R. Ozturk and R. Keskin, 2003. Some physical properties of hemp seed. Biosystems Engineering, 86(2), 213-215.

Sacilik, K., Celik, T. and A. Colak, 2007. Moisture content and bulk density dependence of dielectric properties of safflower seed in the radio frequency range. Journal of Food Engineering, 78: 1111-1116.

Sadeghi, H., F. Khazaei, S. Sheidaei and L. Yari, 2011. Effect of dimension on seed germination behavior of safflower (Carthamus tinctorius L.). ARPN Journal of Agricultural and Biological Science 6(4): 5-8.

Seifi, M. R., R. Alimardani, A. Akram and A. Asakereh, 2010. Moisture-depend physical properties of safflower (Goldasht). Adv. J. Food Sci. Technol., 2(6): 340-345.

Singh, V. and N. Nimbkar, 2007. Safflower (Carthamus tinctorius L.). In: Singh, R. J. (Ed.). Genetic Resources, Chromosome Engineering and Crop Improvement : Oilseed
Crops, Vol. 4, CRC, Boca Raton, FL 33487-7742, USA, pp. 167-194.

Singh, V., N. M. Kolekar and N. Nimbkar, 2008. Breeding strategy for improvement of flower and seed yields insafflower. Proceedings of the 7th International Safflower Conference, Wagga Wagga, New South Wales, Australia.

Tarighi, J., Mohtasebi, S.S. and Mahmoodi, A., 2010. Effect of moisture content on some physical properties of safflower (var. Darab) seeds. Journal of Food, Agriculture \& Environment, 8(3-4): 602-606 .

Tarighi, J., A. Mahmoudi and M.K. Rad, 2011. Moisturedepend some postharvest properties of two varieties of safflower (Darab and Goldasht). Modern Applied Science 5(1): 139-148.

Urie, A.L., 1986. Inheritance of paritial hull in safflower. Crop Sci., 26: 493-498.

Urie, A. L. and D. E. Zimmer, 1970. A reduced-Hull seed character of safflower. Crop Sci., 10: 371-372.

Uter, J., 2008. Safflower in European floriculture: a review. Proceedings of the 7th International Safflower Conference, Wagga Wagga, New South Wales, Australia.

Uysal, N., H. Baydar and S. Erbas, 2006. Determination of agricultural and technological properties of safflower (Carthamus tinctorusL.) lines developed from Isparta population. Suleyman Demirel University Journal of the Faculty of Agriculture, 1(1):52-63.

Vorpsi, V., F. Harizaj, N. Bardhi, V. Vladi and E. Dodona, 2010. Carthamus tinctorius L., the quality of safflower seeds cultivated in Albania. Research Journal of Agricultural Science, 42 (1): 326-331.

Weiss, E.A., 2000. Safflower. In: Oilseed Crops, Blackwell Sci. Ltd., Victoria, Australia, pp 93-129.

Yadava, D. K., S. Vasudev , N. Singh, T. Mohapatra and K.V. Prabhu, 2012. Breeding Major Oil Crops: Present Status and Future Research Needs. S.K. Gupta (ed.), In: Technological Innovations in Major World Oil Crops, Chapter 2, Volume 1: Breeding. 